\title{
Intraphrasale Permutation innerhalb der nominalen Initialphrase: ein deutsch- polnischer Vergleich
}

\begin{abstract}
Im Beitrag wird die nominale Initialphrase im Deutschen und im Polnischen im Hinblick auf die intraphrasale Permutation der Bestandteile verglichen. Da die Variabilitätsskala in dem zu untersuchenden Bereich im Polnischen (im Vergleich zum Deutschen) größer ist, ist in der Diskussion von der Perspektive des Polnischen auszugehen. An stellungsmäßig parallelen deutschen Beispielen wird beleuchtet, wo sich die deutsche Nominalphrase beim Verhältnis von Hierarchie und Abfolge von Stellungselementen in der internen Struktur der Nominalphrase deutlich abhebt. Es werden Faktoren überprüft, die in den beiden Sprachen im umrissenen Bereich mehrere, für das Ohr des Muttersprachlers der jeweiligen Sprache noch akzeptable Anordnungsfolgen motivieren.
\end{abstract}

Abstract (pol.): W artykule porównuję możliwości permutacji elementów wewnątrz niemieckiej i polskiej frazy nominalnej znajdującej się w pozycji inicjalnej zdania. Ponieważ skala permutacji w badanym obszarze jest większa w języku polskim niż niemieckim, przyjmuję w dyskusji perspektywę języka polskiego. Za pomocą analogicznych niemieckich przykładów pokazuję zasadnicze różnice w szyku i hierarchii komponentów wewnątrz niemieckiej frazy nominalnej. Wskazuję na czynniki, które w obu językach motywują poszczególne przesunięcia wewnątrzfrazowe, szczególnie te, które pozostają na granicy akceptowalności.

\section{Zielsetzung}

Der Aufsatz verfolgt das Ziel, die nominale Initialphrase im Deutschen und im Polnischen im Hinblick auf die intraphrasale Permutation der Bestandteile zu vergleichen. In beiden Sprachen sind die Funktionen der Bestandteile der Nominalphrase an die Position in der internen Phrasenstruktur gebunden. Auch wenn die jeweiligen Bestandteile phrasenintern syntaktischen Hierarchieregeln unterliegen, gibt es Abfolgevariationen. Sie sind im Deutschen nur auf einige wenige Möglichkeiten beschränkt, im Polnischen hingegen ist mehr möglich.

Die Permutationsmöglichkeiten in der internen Struktur der polnischen Nominalphrase stehen in vergleichsweise vielen Fällen im direkten Verhältnis 
zur angestrebten Informationsverteilung im Satz (vom Allgemeinen zum Besonderen): Steht im Polnischen die Nominalphrase satzinitial, können Attribute des Kernnomens kontext- und intonationsbedingt sogar häufiger permutieren und sind zudem in ihrer Abfolge in höchstem Maße variabel. Die Schiebemöglichkeiten in der Initialposition erscheinen auf Anhieb als limitiert und regelrecht: Stehen die Attributelinks des Kernnomens, so markieren sie den neutralen Anschluss an den vorangehenden oder nachfolgenden Kontext. Rechts des Kernnomens dienen sie in der Tendenz der Hervorhebung. Durch intonatorische Mittel kann dies aber immer aufgehoben werden, indem der Fokus beliebig verlegt werden kann. Ob dabei jede Abfolge als akzeptabel oder als nicht akzeptabel erscheint, bleibt dahingestellt (ausführliche Diskussion unter Kap. 3).

Da die Variabilitätsskala in dem zu untersuchenden Bereich im Polnischen (im Vergleich zum Deutschen) größer ist, ist in der Diskussion von den polnischen Beispielen auszugehen. An stellungsmäßig parallelen deutschen Beispielen wird zunächst allgemein beleuchtet, wo sich die Nominalphrase beim Verhältnis von Hierarchie und Abfolge von Stellungselementen in der internen Struktur der Nominalphrase deutlich abhebt. Es wird der Frage nachgegangen, ob zwischen den beiden Sprachen im umrissenen Bereich tatsächlich Unterschiede in der Möglichkeit bestehen, mehrere, für das Ohr des Muttersprachlers der jeweiligen Sprache noch akzeptable Anordnungsfolgen zu produzieren. Als kontrastives Untersuchungsfeld setzt sich die Nominalphrase aus folgenden Bestandteilen zusammen: ${ }^{1}$

(Det. $)_{\text {u.a. dt./seltener poln. }}+($ Adj. $),\left(\mathrm{NP}_{\text {im Gen. }}\right)$ Nomen $\left(\mathrm{NP}_{\text {im Gen. }}\right),(\text { Adj. })_{\text {u.a. poln./selten dt. }}$

Das Kernnomen nimmt das Zentrum der gesamten Nominalphrase ein; das Determinativ eröffnet die Nominalklammer, die das Kernnomen schließt; um das Kernnomen herum können weitere Attribute angeordnet sein. In solchen Fällen ist die Linearstruktur einer polnischen und einer deutschen Nominalphrase gleich. Allerdings sind im Polnischen Determinative generell nicht so häufig wie im Deutschen; Adjektive in der postnominalen Position sind im Deutschen nur begrenzt zulässig, während sie im Polnischen der Normalfall sind. Genitivattribute stehen sowohl vor als auch hinter dem Kopfnomen, wobei im Deutschen eine Regel festzustellen ist, Personenbezeichnungen, Personennamen und (seltener) andere Eigennamen (als sächsischer Genitiv) vor das Kopfnomen zu stellen.

1 Zur Erläuterung: Die vorgeschlagene abstrakte Darstellung beinhaltet alle potenziellen Positionen, die sich aber gegenseitig blockieren können. 
Auf die Anordnungsmuster für die obigen Typen der Nominalphrase im Kontrast wird unter Kapitel 2 eingegangen werden.

Eines kurzen Kommentars bedürfen hier der Terminus Initialphrase und die Beschränkung der Analyse auf die (nominale) Initialphrase. Als Initialphrase des Satzes fungiert in meinem Verständnis diejenige Phrase, sei sie einwortig (pro) nominal, durch eine Wortgruppe oder durch einen Nebensatz beliebiger Länge bzw. durch eine Infinitivkonstruktion repräsentiert, die in der Grundstruktur des Satzes als obligatorische Konstituente der Satzbasis (= Stellungsglied) direkt an deren Anfang (und vor dem finiten Verb) steht. Den Terminus Initialphrase übernehme ich von Cirko (2012: 130-131), der hierfür eine Unterscheidung zwischen dem Initialwort (als dem ersten Wort in der Äußerung) und der Initialphrase (als der ersten Phrase in der Äußerung in der Funktion des Stellungsgliedes) bietet. In Bezug auf das Initialwort spricht er von der ,absoluten Initialposition“ (= Vor Millionen von Jahren wuchs hier ein Urwald., ebd.: S. 131; die Hervorhebung durch Unterstreichung von E.B.), im zweiten Fall von der „erweiterten Initialposition“ (= Vor Millionen von Jahren wuchs hier ein Urwald., ebd.; die Hervorhebung von E.B.). Ich gehe davon aus, dass Attribute und Prädikatsteile, die in der Initialposition des Satzes stehen können und auch als Stellungselemente fungieren, aber syntaktisch nur Satzgliedteile (also nicht autonom) sind, dem obigen Definitionsvorschlag nach eher dem Initialwort zuzurechnen wären. Der Gefahr einer anders gelagerten Diskussion möchte ich von vorneherein entgehen und beschränke die Anforderungen an meine Untersuchung derart, dass sich eine Restriktion in der Berücksichtigung der linearen Gesamtstruktur der Initialphrase ohne Dislozierungen zeigt.

Die obige Beschränkung erweist sich für Darstellungszwecke der phraseninternen Permutation auch deshalb als nützlich, da es in beiden Sprachen einige regelhafte Typen von dislozierbaren Attributen des Nomens gibt, die sich um das finite Verb herum anordnen, genauer gesagt, nach dem finiten Verb, also auch außerhalb der linearen Struktur der Nominalphrase stehen können, wie etwa Alle Bücher hier sind zu verkaufen. ('Wszystkie książki tu są do sprzedania.') $\rightarrow$ Die Bücher hier sind alle zu verkaufen. ('Książki tu są wszystkie do sprzedania.'). Die Umstellproben in Kapitel 3 betreffen nur eine kontinuierlich realisierte Nominalphrase ohne Berücksichtigung der Möglichkeiten ihrer Spaltung bzw. der in Distanz stehenden Attribute. Meine Überlegungen beschränken sich auf die Permutation im Inneren der Nominalphrase in der Initialposition des deutschen Verbzweitsatzes und des polnischen Satzes mit dem finiten Verb an zweiter Stelle des Aussagesatzes. 


\section{Die Anordnung der Stellungselemente der Nominalphrase im Kontrast}

Der Kern der Nominalphrase besteht in beiden Sprachen immer nur aus einem Wort - dem Nomen. Daraus ergeben sich in der Nominalphrase je zwei Stellungsfelder um das Nomen herum. Hierfür ist m.E. die polnische Terminologie bezüglich der Topologie des Satzes: linkes und rechtes Feld ${ }^{2}$ besonders bequem zu handhaben. Aufgrund der Ausdrucksformen der Bestandteile der Nominalphrase gibt es prinzipiell keine Unterschiede im Deutschen und im Polnischen, bis auf den kontrastkonstitutiven Typ der instrumentalen Nominalphrase im rechten Feld der polnischen Nominalphrase, die im Deutschen als genitivische oder präpositive Nominalphrase realisiert werden kann, z.B.:

$\underline{\text { zarzadzanie przedsiębiorstwem }}_{(\mathrm{NP} \text { Instr.) }} \rightarrow$ die Verwaltung des Unternehmens ${ }_{(\mathrm{NP} \text { Gen.) }}$

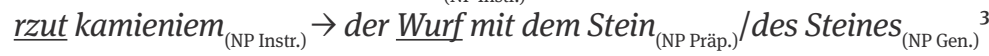

Die Art und die Zahl der Bestandteile der Nominalphrase machen sie aber für die kontrastive Betrachtung weniger interessant. ${ }^{4}$ Eine Reihe markanter Unterschiede ergibt sich unter anderem aus dem Vergleich der Position der Bestandteile im Verhältnis zum nominalen Kern der Phrase und ihrer Beweglichkeit um den Kern herum. Schauen wir uns zunächst eine Nominalphrase an, deren rech-

2 In einer sprachspezifischen Satztopologie wird mit der Bezeichnung linkes Feld der Satzbereich verstanden, der sich links von der finiten Verbform befindet. Er entspricht dem linken Außenfeld, Vorvorfeld und dem Vorfeld im Deutschen. Den Verhältnissen in der Nominalphrase angepasst, ist das linke Feld ein Stellungsbereich, der sich links vom Phrasenkopf befindet. Das für das Polnische topologisch definierte Stellungsfeld nach dem Finitum bezeichnen die Grammatiken als rechtes Feld. Dieser Satzbereich entspricht dem Mittelfeld, dem Nachfeld und dem rechten Außenfeld im Deutschen. In der Nominalphrase ist es ein Bereich rechts des nominalen Phrasenkopfs.

3 Beispiele von Engel et al. (1999: 918, 929). Unterschiede hinsichtlich der Morphosyntax zwischen deutschen und polnischen nominalen Kasusattributen resultieren vor allem aus dem differenten Kasussystem beider Sprachen. Zu Typen von Nominalphrasen im Polnischen vgl. Grochowski/Karolak/Topolińska (1984: 330), im deutsch-polnischen Vergleich vgl. Engel et al. (1999: 918ff.) und Urbaniak-Elkholy (2014: 96ff.).

4 Ein unabänderliches, gut untersuchtes Faktum ist z.B., dass das polnische Nomen viel häufiger als das deutsche ohne besetztes linkes Feld vorkommt, auf jeden Fall häufig ohne Determinativ (s. das Schema der Nominalphrase in Kap. 1). Determinative sind im Polnischen nicht in derselben Art wie im Deutschen regelmäßige Begleiter des Nomens; vgl. Witwicka-Iwanowska (2012). 
tes Feld durch eine genitivische Phrase (aus dem Determinativ und dem Nomen) besetzt ist:

$$
\begin{aligned}
& \text { (Det.) }{ }_{\text {nur dt. Nomen }} \text { (NP im Gen. aus: Det. + Nomen) } \\
& \begin{aligned}
\underline{\text { mama }} & \text { mojej } \underline{\text { mamy }} \\
\text { die } \underline{\text { Mutter }} & \text { meiner } \underline{\text { Mutter }}
\end{aligned}
\end{aligned}
$$

Diese Nomenangabe in Form des genitivus possessivus bringt ein Zugehörigkeitsverhältnis zwischen zwei Größen zum Ausdruck; sie wird in beiden Sprachen in

\begin{tabular}{|c|c|}
\hline$\underline{\operatorname{mama}}_{\mathrm{Nom} .}$ mojej $\underline{\text { mamy }}_{\mathrm{Gen} .}$ & die $\underline{\text { Mutter meiner } \underline{\text { Mutter }}}$ \\
\hline mama $_{\mathrm{Nom} .} \underline{\text { mamy }}_{\mathrm{Gen} .}$ mojej & ${ }^{\star}$ die Mutter Mutter meiner \\
\hline $\begin{array}{l}\text { mamy }_{\text {Gen. }} \text { mojej } \underline{\operatorname{mama}}_{\mathrm{Nom} .} \\
\text { mojej } \underline{\operatorname{mamy}}_{\mathrm{Gen} .} \underline{\operatorname{mama}}_{\mathrm{Nom} .}\end{array}$ & $\begin{array}{l}{ }^{\star} \text { Mutter } \text { meiner }_{\text {Gen. }} \frac{\text { Mutter }_{\text {Nom. }} \text { /aber: Mutters } \underline{\text { Mutter }}}{\text { ?meiner } \underline{\text { Mutter Mutter }} \text { /aber: Mutters } \underline{\text { Mutter }}}\end{array}$ \\
\hline
\end{tabular}
der Regel dem Nomen nachgestellt. Ich verschiebe die Stellungselemente in beiden Sprachen:

Im Unterschied zum Deutschen kann ein attributiver Genitiv im Polnischen beinahe immer eine beliebige Position beziehen, ohne negative Folgen für die Grammatikalität der Nominalphrase zu haben. Im Deutschen wird der Genitiv meist nachgestellt. Steht er voran, so nimmt er die Stelle des Determinativs ein und ist mit diesem nicht kompatibel. Aus dem Vergleich der Schemata ergibt sich auch, dass das (hier: possessive) Determinativ im Polnischen in beiden Feldern erscheint und es auch mit dem Phrasenkopf in beiden Feldern in Kasus, Numerus und Genus kongruiert. Auch wenn Determinative bei Nachstellung im Ohr eines nativen Sprechers etwas hervorgehoben oder archaisch wirken mögen, bietet das Polnische hier im Unterschied zum Deutschen ein breiteres Inventar von Determinativen, das sich spontan und frei fortsetzen lässt, ohne als ungrammatisch betrachtet werden $\mathrm{zu}$

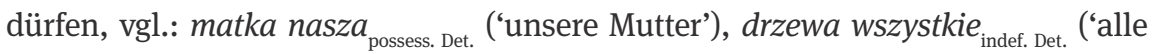

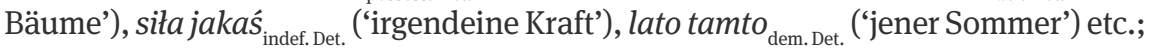
vereinzelte deutsche Beispiele für den analogen Gebrauch der Determinative verzeichnen z.B. Engel et al. (1999: 925): Vater unser, Kindlein mein (Volkslied).

Nehmen wir nun die Nominalphrase mit einem attributiven Adjektiv:

$$
\begin{array}{lll}
(\text { Det. })+ & (\text { Adj. }) & \text { Nomen }(\text { Adj. })\left(+ \text { Det. }_{\text {nur poln. }}\right) \\
\text { jakaś } & \text { tadna } & \underline{\text { sukienka }}
\end{array}
$$

5 Meiner Mutter Mutter, meiner Mutter Auto etc. sind etwas veraltet, aber auf jeden Fall möglich und poetisch. 


\begin{tabular}{|c|c|c|}
\hline \multicolumn{2}{|c|}{ ein schönes } & $\underline{\text { Kleid }}$ \\
\hline jakaś & & sukienka ładna \\
\hline *ein & & Kleid schönes \\
\hline & & sukienka ładna jakaś \\
\hline & & * Kleid schönes ein \\
\hline & & sukienka jakaś ładna \\
\hline & & * Kleid ein schönes \\
\hline
\end{tabular}

Die rechte Position des attributiven Adjektivs im Verhältnis zum nominalen Kern macht den interessanten sprachspezifischen Kontrast aus. ${ }^{6}$ Im Polnischen haben Adjektive größere Stellungsfreiheiten, die ihrerseits Bedeutungsnuancierungen zur Folge haben (können); manchmal dient aber die Verschiebung ins eine oder andere Feld nur der Anpassung an den Kontext. So ist nach einer Bedeutungsverschiebung in folgenden Beispielen vergeblich zu suchen: $\dot{z}$ ótty ${ }_{\mathrm{Adj}}$. kwiatek/kwiatek $\dot{z}$ ótty ${ }_{\text {Adj. }}$ ('gelbe Blume'), niebieskie ${ }_{\text {Adj. }}$ niebo/niebo niebieskie ${ }_{\text {Adj. }}$ ('blauer Himmel'), drewniany $_{\text {Adj. }}$ stót/stót drewniany ${ }_{\text {Adj. }}$ ('Holztisch'). Wenn Bedeutungsnuancierungen mitschwingen, so charakterisieren Adjektive bei der Voranstellung prinzipiell den akzidentellen bzw. vorläufigen Charakter einer Eigenschaft, bei der Nachstellung eine wesenhafte bzw. konstitutive Eigenschaft (vgl. Grochowski/Karolak/ Topolińska 1984: 384; Engel et al. 1999: 921, 925-926; Nagórko 2005: 261, 283-288): aktor komiczny $_{\text {Adj. }}$ ('Spieler komischer Rollen') / komiczny ${ }_{\text {Adj. }}$ aktor (Bewertung:

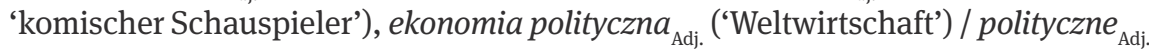
zachowanie (Bewertung: 'höfliches Verhalten'), panna mtoda $a_{\text {Adj }}$ ('Braut') / mtoda $a_{\text {Adj. }}$ panna (Beschreibung/Bewertung: 'eine junge Dame').

$\mathrm{Zu}$ diesem Anordnungsmuster ist ein Kommentar zu den adjektivisch gebrauchten Partizipien (als Attribute) nötig, da sie morphosyntaktisch attributiven Adjektiven ähneln. Es gibt im Polnischen offenbar eine Tendenz, attributive Partizipien zunehmend nachzustellen, die aber m.E. eher intuitiv erkannt als fachlich untermauert werden kann: Adjektivisch gebrauchte Partizipien (als Attribute) im rechten Feld schränken den semantischen Geltungsbereich des Nomens ein, vgl. czlowiek palacy Partizip $\rightarrow$ palacz ('Raucher') vs. palacy Partizip $_{\text {(papierosa; tutaj }}$ etc.) człowiek (stärkere Betonung der Tätigkeit: 'ein (Zigarette; hier etc.) rauchender

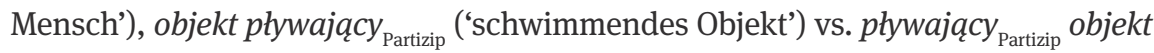
(stärkere Betonung der Tätigkeit: 'schwimmendes Ding/Objekt') etc. Ihre Position

6 Die beiden letztgenannten Beispiele aus dem Polnischen nach dem Schema: sukienka ładna jakaś (wortwörtl.: „Kleid schönes ein“) und sukienka jakaś ładna (wortwörtl.: „Kleid ein schönes“) zeigen darüber hinaus, dass die „Wanderung“ des Determinativs um das nachgestellte Adjektiv herum möglich ist; im Deutschen ist sie hingegen inakzeptabel. $\mathrm{Zu}$ allen Umstellsequenzen siehe Kapitel 4. 
links oder rechts des Bezugswortes kann wohl auch ohne erkennbare Bedeutungsänderung verbunden sein, vgl.: tańcząca $a_{\text {Partizip }} d z i e w c z y n a / d z i e w c z y n a$ tańcząca $a_{\text {Partizip }}$

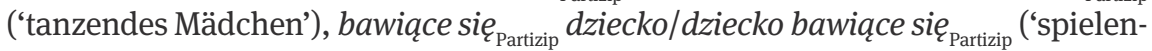
des Kind'). Morphosyntaktisch ist auffallend, dass das Adjektiv und das adjektivisch gebrauchte Partizip im Polnischen in beiden Feldern mit dem Nomen kongruieren.

Adäquate Übersetzungen obiger polnischer Beispiele stellen - den deutschen Verhältnissen angepasst - Adjektive (als Attribute) dem regierenden Nomen voran (abgesehen von deutschen Komposita, die auch als Entsprechungen polnischer Nominalphrasen in Frage kommen). Stehen attributive Adjektive im Deutschen nach dem Nomen, sind sie unflektiert oder noch teilweise flektiert und gehören meistens der poetischen oder gehobenen Sprache an (so wie bei Röslein rot). Es gibt zwar auch aktuelle Fälle mit nachgestellten Adjektiven wie etwa Schauma mild, Eiche rustikal, Forelle blau; die deutschen Grammatiken behandeln diese Konstruktionen aber unterschiedlich. Bei Typen wie Karpfen blau, Forelle blau setzen Zifonun/Hoffmann/Strecker (1997: 1991) eine elliptisch verwendete Adverbialkonstruktion und Eisenberg (2006: 237) einen idiomatisierten Ausdruck an; Typen wie Schauma mild, Henkel trocken grenzt Eisenberg (ebd.) dabei als Produktbezeichnungen ein, was zu der Annahme verleiten kann, es handle sich nicht um eine im Deutschen übliche Attribuierung. Bei Typen wie ihr Lächeln, halb geheimnisvoll, halb spöttisch setzen Engel et al. (1999: 1235) einen kategoriell anderen Status, nämlich den Status der Apposition, an. Unflektierte Adjektive sowie adjektivisch gebrauchte Partizipien, die dem Nomen als lockerer Nachtrag folgen und (meist) in Relativsätze umgebaut werden können, werden in der Duden-Grammatik (2009: 346-347) als satzwertige Adjektiv- bzw. Partizipphrasen angesehen (so wie bei Die Sekretärin, müde und abgespannt, legt die Füße auf das Pult). Lockere Nachträge mit flektierten Adjektiven wie Kinder, auch kleinere, merken sehr wohl, wo Grenzen liegen werden in der Duden-Grammatik (2009: 343) als elliptische Nominalphrasen ${ }^{7}$, d.h. auch als lockere Appositionen aufgefasst. Damit gelten die Möglichkeiten der Verschiebung von Adjektiven nach rechts als ausgeschöpft.

Aus dem Vergleich der Stellungsregeln und -tendenzen ergeben sich interessante Einsichten in die Mehrfachbesetzung mit attributiven Adjektiven. Im Polnischen verursacht die Mehrfachsetzung eine Verteilung der Adjektive auf das linke und rechte Feld, wobei zum rechten Feld Adjektive tendieren, die, wie oben angeführt, eine wesenhafte oder immanente Bedeutung ausdrücken. Daher kann

7 Die Umschreibung dieses Satzes ist: Kinder, und zwar auch kleinere Kinder, merken sehr wohl, wo Grenzen liegen. 
diese Verteilung in der Regel der bedeutungsdifferenzierenden Hierarchisierung der Eigenschaften einer nominalen Größe dienen, vgl.: podstawowe ${ }_{\text {Adj. }}$ prawa pracownicze $_{\text {Adj. }}$ ('die grundlegenden Arbeiterrechte/die grundlegenden Rechte der Arbeiter') - das Adjektiv pracownicze bestimmt das Nomen (als Kopf der Phrase) näher, das vorangestellte Adjektiv hingegen die ganze Nominalphrase aus Nomen und nachgestelltem Adjektiv; so ähnlich auch in: europejsk $a_{\text {Adj. }}$ literatura wspótczesna $_{\text {Adj. }}$ ('die zeitgenössische europäische Literatur' - es handelt sich hier um die zeitgenössische Literatur mit europäischem Schwerpunkt), niski $i_{\text {Adj }}$ stót drewniany $_{\text {Adj. }}$ ('der kleine Tisch aus Holz' - dies ist ein Holztisch, der klein ist) etc. Im Deutschen führt die Mehrfachsetzung durch attributive Adjektive zu einer pränominalen Häufung. ${ }^{8}$

\section{Die nominale Initialphrase: intraphrasale Permutation}

Für die Zwecke der kontrastiven Betrachtung der Permutationen innerhalb der initialen Nominalphrase wähle ich die Phrase aus bestimmtem Determinativ, Adverb und Adjektiv (in Voranstellung); sie bildet das Subjekt des Satzes. Das Subjekt hat in beiden Sprachen gegenüber anderen Satzgliedern den Vorzug bei der Thematisierung: Eine als Subjekt fungierende Nominalphrase, die etwas Bekanntes bezeichnet, behält in beiden Sprachen im Allgemeinen den Platz an der Spitze des Satzes gegenüber anderen bekannten Satzgliedern. Das Subjekt im Polnischen hat eine starke Linkstendenz: Kein anderes Stellungsglied kann dem Subjekt in dieser Beziehung gleichen. Zudem ist im Polnischen (im deutlichen Kontrast zum Deutschen) die Initialposition, besetzt durch die Nominalphrase, eine besonders „permutationssensitive Stelle“, wobei vor allem informationsstrukturell-kommunikative und stilistische Gründe, kaum aber grammatische Regeln ins Gewicht fallen. Im Zuge der Permutationen in der Initialposition entsteht ein Sprachfaktum, das zur Charakterisierung mancher Permutationen in der spontanen Rede als (spontane) Einmaligkeit führen kann. Die (analogen)

\footnotetext{
8 Die Anzahl der attributiven Adjektive in dieser Position kann so gut wie unbegrenzt sein, doch auch sie unterliegen in der Abfolge genauen semantischen Regularitäten. Alle Adjektive sind dabei in der Linearstruktur der Nominalphrase syntaktisch gleichartig, d.h., sie beziehen sich in gleicher Weise auf den nominalen Kern; die semantische Klassifikation wird als für die Problemstellung dieses Aufsatzes nicht relevant außer Acht gelassen. Zur Grundanordnung der attributiven Adjektive im pränominalen Bereich der deutschen Phrase vgl. z.B. Eroms (2000: 269), bei Anwendung der Terminologie von Eichinger (1991); dort auch weitere Literaturhinweise.
} 
nicht-initialen Phrasen werden hingegen mehr präzise strukturiert und Stellungstendenzen in diesen Phrasen viel strenger befolgt. Als Annahme gilt: Die Umstellproben, die ich in der internen Phrasenstruktur am Anfang des Satzes weiter unten vornehmen werde, beeinflussen die angenommene Thema-RhemaProgression im Satz nicht.

Ta bardzo dobra książka wcale nie chciała dobrze się sprzedawać. 'Dieses sehr gute Buch wollte sich gar nicht gut verkaufen.'

Die folgenden Annahmen gelten zur Charakterisierung dieser Nominalphrase: Ihrer Determination nach ist ihr Referent bekannt; das adjektivische Attribut bestimmt das regierende Nomen näher und trägt so zur Bedeutung der ganzen Nominalphrase bei; beide Elemente (Determinativ, Adjektiv) kongruieren mit dem Nomen in Kasus und Numerus (Flexionsmarker bei beiden Elementen). Das Adverb modifiziert das Adjektiv und ist grammatisch gesehen vollkommen fakultativ. Im Folgenden soll bei den Umstellproben das Verhalten der Konstituenten in beiden Sprachen gezeigt werden. Wenn die Probe eine nicht korrekte Phrase (bis hin zum nicht korrekten Satz) ergibt, wird sie mit * markiert; bei noch möglichen, aber fraglichen Fällen steht ein Fragezeichen (ein Kommentar zu diesen Fällen folgt weiter unten).

Ta książka bardzo dobra (...).

Ta książka dobra bardzo (...).

*Ta dobra ksiazzka bardzo (...).

*Ta bardzo ksiażka dobra (...).

Książka ta bardzo dobra (...).

Książka ta dobra bardzo (...).

* Książka bardzo ta dobra (...).

* Książka dobra ta bardzo (...).

${ }^{*}$ Książka bardzo dobra ta (...).

*Książka dobra bardzo ta (...).

?Bardzo dobra ta ksiażka (...).

?Bardzo dobra książka ta (...).

*Bardzo ta dobra ksiażka (...).

*Bardzo ta książka dobra (...).

*Bardzo ksiązka ta dobra (...).

* Bardzo ksiażka dobra ta (...).

?Dobra bardzo ta ksiązka (...).

?Dobra bardzo książka ta (...).

*Dobra ta bardzo książka (...).
${ }^{*}$ Dieses Buch sehr gute (...).

${ }^{*}$ Dieses Buch gute sehr (...).

${ }^{*}$ Dieses gute Buch sehr (...).

${ }^{*}$ Dieses sehr Buch gute (...).

${ }^{*}$ Buch dieses sehr gute (...).

${ }^{*}$ Buch dieses gute sehr (...).

${ }^{*}$ Buch sehr dieses gute (...).

${ }^{*}$ Buch gute dieses sehr (...).

${ }^{*}$ Buch sehr gute dieses (...).

${ }^{*}$ Buch gute sehr dieses (...).

*Sehr gute dieses Buch (...).

${ }^{*}$ Sehr gute Buch dieses (...).

${ }^{*}$ Sehr dieses gute Buch (...).

${ }^{*}$ Sehr dieses Buch gute (...).

*Sehr Buch dieses gute (...).

*Sehr Buch gute dieses (...).

${ }^{*}$ Gute sehr dieses Buch (...).

${ }^{*}$ Gute sehr Buch dieses (...).

${ }^{*}$ Gute dieses sehr Buch (...). 
${ }^{*}$ Dobra ta ksiażka bardzo (...).

${ }^{*}$ Dobra ksiażka ta bardzo (...).

${ }^{*}$ Dobra książka bardzo ta (...).
${ }^{*}$ Gute dieses Buch sehr (...).

${ }^{*}$ Gute Buch dieses sehr (...).

${ }^{*}$ Gute Buch sehr dieses (...).

Das Umstellresultat zeigt eine gewisse Wortstellungsfreiheit im Inneren der polnischen Phrase, gar keine in der deutschen Phrase. Im Deutschen sind aufgrund der früher möglichen Stellungsfreiheiten die adjektivischen Nachstellungen noch vorfindbar, wenn auch wohl nicht immer akzeptabel, vgl. etwa: 'Dieses Buch, sehr gut, wollte sich gar nicht gut verkaufen. ${ }^{9}$ Eine solche nominale Fügung erfordert aber das unflektierte nachgestellte Adjektiv, und sie könnte auch als eine elliptisch verwendete Prädikativkonstruktion interpretiert werden wie etwa: Dieses Buch - es ist sehr gut - wollte sich gar nicht gut verkaufen. Für das Polnische hingegen - es sei betont: v.a. in der mündlichen Kommunikation, wo Stellung von Elementen und Intonation zusammenspielen - ergeben sich im Umstellresultat vier akzeptable Sätze:

Ta ksiażka bardzo dobra wcale nie chciała dobrze się sprzedawać. Ta książka dobra bardzo wcale nie chciała dobrze się sprzedawać. Ksiażka ta bardzo dobra wcale nie chciała dobrze się sprzedawać. Ksiażka ta dobra bardzo wcale nie chciała dobrze się sprzedawać.

Die reguläre, stilistisch neutrale Grundfolge der Attribute im linken Feld der polnischen Nominalphrase lässt die folgende Anordnung erwarten: Determinativ Zahladjektiv - (eventuell Adverb) - Adjektiv. Die Nachstellungen der Elemente Adverb + flektiertes Adjektiv (wie hier: bardzo ('sehr') + dobra ('gut')) sind im Polnischen an sich nichts Ungewöhnliches. Doch ist hier zu vermuten, dass solche Fügungen häufiger und auch natürlicher in der gesprochenen Sprache vorkommen. Allgemein hängt es von stilistischen Präferenzen ab, welche Phrasenteile wie betont werden; für solche Abfolgealternativen fungieren paralinguistische Mittel als Unterstützung, z.B. das Sprechtempo, die Klangfärbung oder allgemein die Artikulationsart. Sie treten zwar immer in Verbindung mit der Sprache auf, aber sie gehören nicht im strengen Sinn zur Sprache. Eine Realisierung könnte so aussehen (die Hervorhebung wird halbfett markiert):

Ta ksiażka (Pause) bardzo dobra (Pause) wcale nie chciała dobrze się sprzedawać. Ta książk (Pause) dobra bardzo (Pause) wcale nie chciała dobrze się sprzedawać.

9 Man könnte sich bei Betonung des sehr gut und mit gebührenden Sprechpausen, diesen Satz in der gesprochenen Sprache vorstellen. Dann liegt das Gewicht, die Betonung auf sehr gut, wodurch auch der Sprecher in gewissem Sinne charakterisiert wird: als dominanter Sprecher. 
Książka ta (Pause) bardzo dobra (Pause) wcale nie chciała dobrze się sprzedawać. Książka ta (Pause) dobra bardzo (Pause) wcale nie chciała dobrze się sprzedawać.

In den zwei ersten Varianten suggerieren die Umstellung und die Betonung eine konzessive Implikation zwischen der Eigenschaft des Buches und dem Geschehen: Ta książka, pomimo że/chociaż bardzo dobra, wcale nie chciała dobrze się sprzedawać. (= obwohl-Satz). Das nachgestellte Demonstrativum książka ta ('Buch dieses') ist in den zwei letzten Varianten deutlich rückverweisend: „das schon erwähnte Buch“. Hier könnte man auch an ein stilistisch zu erklärendes Weglassen des unbetonten książka-Lexems denken: Ksiażka ta, bardzo dobra książka, wcale nie chciała dobrze się sprzedawać.

Vier weitere Beispiele sind nur noch unter Umständen akzeptable Umstellungen; kontextfrei betrachtet können sie wohl seltsam erscheinen:

?Bardzo dobra ta książka (Pause) wcale nie chciała dobrze się sprzedawać. ?Bardzo dobra książka ta (Pause) wcale nie chciała dobrze się sprzedawać. ?Dobra bardzo ta ksiażka (Pause) wcale nie chciała dobrze się sprzedawać. ?^Dobra bardzo książka ta (in einem Atemzug ausgesprochen; bei emphatischer Redeweise) wcale nie chciała dobrze się sprzedawać.

Durch die gemeinsame Versetzung des adjektivischen Attributs mit dem Adverb (bardzo dobra ('sehr gut')/dobra bardzo ('gut sehr')) in das linke Feld der Nominalphrase wird die Eigenschaft des Subjekts (książka/Buch) nachdrücklich hervorgehoben. Kommunikativ gesehen sind die Beispiele nur akzeptabel, wenn sie etwa durch paralinguistische Mittel zusätzlich unterstützt werden. Zwar scheint grammatisch eine solche Aufspaltung der Nominalphrase im Polnischen überhaupt nichts Ungewöhnliches zu sein, die obigen Beispiele gelten aber keineswegs ohne Ausnahme. Sie lassen sich auf spezielle Fälle und eine entsprechende kommunikative Funktion zurückführen. Grochowski/Karolak/Topolińska (1984: 384) weisen darauf hin, dass die Nachstellungen der Attribute verschieden starke Rhematisierungseffekte mit sich bringen. In Sätzen wie Kwiatk $a_{\text {Nom. }}$ barwnego $_{\text {Adj. }}$.

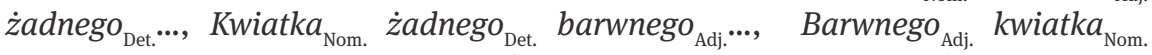
$\dot{z}$ adnego ${ }_{\text {Det. }}$ nie było pod oknami ('es gab *Blume bunte keine/"Blume keine bunte/‘bunte Blume keine vor den Fenstern') dient die Nachstellung einer absichtlichen Archaisierung; die deutschen Stellungsregeln würden die Verschiebung auch in diesem Fall blockieren: ${ }^{\star}$ Blume bunt gab es keine vor den Fenstern. Davon sind solche Sätze zu unterscheiden wie Człowiek, każdy, pragnie ułożyć sobie życie jak najlepiej. ('der Mensch, ein jeder, will ein gutes Leben führen'). Das Attribut wird durch zwei Pausen abgetrennt (durch Kommata in der 
geschriebenen Sprache), wodurch es noch stärker rhematisiert wird; der Nachstellung fehlt der Beigeschmack archaischer Stilisierung.

\section{Schlussfolgerungen}

In der internen Struktur der polnischen Nominalphrase gibt es entschieden mehr Permutationsmöglichkeiten als in der analog gebauten deutschen Nominalphrase. Das im Polnischen viel größere Wirkungsfeld für die Permutationen am Anfang des Satzes (als bei anderen, nicht-initialen Phrasen) wird unterstützt vor allem durch kommunikative und artikulatorische Gründe. Die semantisch-grammatischen Regeln für die lineare Anordnung der Elemente der Nominalphrase spielen im Polnischen eine weitaus kleinere Rolle als im Deutschen. Die beiden Äußerungsmedien aber - die geschriebenen Texte und die gesprochenen Texte - sind wesentlich: Während Stellungsregeln bzw. -tendenzen in den geschriebenen polnischen Texten strenger befolgt werden, können sie in der gesprochenen Sprache wieder aufgelockert werden. Die bei den Umstellproben gewonnenen Erkenntnisse zeigen bestimmte hierarchische Beziehungen in der Nominalphrase, die für das Deutsche streng geregelt sind, während das Polnische in diesem Bereich eben noch gewisse Wortstellungsfreiheiten im Inneren der Phrase zulässt. Im Folgenden fasse ich sie zusammen.

1. Determinative im Deutschen sind enger mit dem Nomen verbunden. D.h., sie sind eines der wichtigsten Elemente im linken Feld der deutschen Nominalphrase, und sie sind in dem Sinn obligatorisch (einschl. Nullartikel), als sie überhaupt den Übergang vom Nomen zur Nominalphrase markieren. Determinative im Polnischen sind keine obligatorischen Komponenten der Nominalphrase. Falls sie verwendet werden, entsteht das interessante Sprachfaktum, dass ihre ziemlich freie „Wanderung“ um den nominalen Kopf herum möglich ist. Falls die Stellen (Det.) und (Adj.) in der polnischen Nominalphrase besetzt sind, können Determinative auch die Position rechts oder links des Adjektivs einnehmen.

2. Diese Fähigkeiten zur „Wanderung“ weisen auch die polnischen Adjektive auf, während der früher auch im Deutschen mögliche Gebrauch der nachgestellten Adjektive oder Determinative heute als veraltet (in festen Formen), unter Umständen als Lehnprodukt zu gelten hat. Im Polnischen kongruiert das Adjektiv mit dem Phrasenkopf im linken und im rechten Feld. Das nachgestellte Adjektiv im Deutschen ist unflektiert.

3. Syntaktisch gesehen sind adjektivische Attribute/Partizipien in beiden Sprachen immer fakultativ in der Struktur der Nominalphrase. Außerdem können 
sie fast unbegrenzt erweitert werden, während die Erweiterungsmöglichkeiten des Determinativs als Attribut (u.a. semantisch) beschränkt sind. Die gehäuften attributiven Adjektive/Partizipien und die Determinative gruppieren sich im Deutschen links des Bezugsnomens, im Polnischen hingegen können sie sich auf beide Bereiche, links und rechts des Nomens, verteilen.

4. Die erwähnten Wortstellungsfreiheiten der Adjektive und Determinative in der polnischen Nominalphrase bedeuten nicht automatisch, dass hier keine Regeln herrschen oder dass alles möglich ist. Wenn sie um den nominalen Kopf „,wandern“, können sich Determinative und Adjektive voneinander trennen, nicht aber Adjektive und Adverbien.

5. Wenn etwas normalerweise im linken Feld der Nominalphrase in geordneter Reihenfolge erscheint und plötzlich nach rechts verschoben wird, muss es dafür Gründe geben. Diese Verschiebung erfolgt meistens zum Zweck einer besonderen Hervorhebung; die verschobenen Elemente sind dann ohnehin intonatorisch hervorgehoben. In solchen Fällen starker Hervorhebung spielen „Normalitätsgrad“, gleichzusetzen mit einem „korrekt-sein-Gefühl“, und Intonation ein Wechselspiel. Prosodische Mittel scheinen im Polnischen viel deutlicher als im Deutschen zu interagieren.

\section{Literatur}

Cirko, Lesław (2012): Blockaden der Initialposition im Deutschen und im Polnischen. In: Cortès, Colette (Hg.): Satzeröffnung. Formen, Funktionen, Strategien. Tübingen: Stauffenburg. 129-141.

Duden (2009): Die Grammatik. Unentbehrlich für richtiges Deutsch. Bd. 4. 8., völlig neu erarb. u. erw. Aufl. Mannheim u.a.: Dudenverlag.

Eichinger, Ludwig M. (1991): Ganz natürlich - aber im Rahmen bleiben. Zur Reihenfolge gestufter Adjektivattribute. In: Deutsche Sprache 19. 321-329.

Eisenberg, Peter (2006): Grundriss der deutschen Grammatik. Bd. 2: Der Satz. 3., durchges. Aufl. Stuttgart/Weimar: J.B. Metzler.

Engel, Ulrich et al. (1999): Deutsch-polnische kontrastive Grammatik. Heidelberg: Groos. Eroms, Hans-Werner (2000): Syntax der deutschen Sprache. Berlin/New York: de Gruyter. Grochowski, Maciej/Karolak, Stanisław/Topolińska, Zuzanna (1984): Gramatyka współczesnego języka polskiego. Bd. 1: Składnia. Warschau: PWN.

Nagórko, Alicja (2005): Zarys gramatyki polskiej. Warschau: PWN.

Urbaniak-Elkholy, Magdalena (2014): Komplexe deutsche Nominalphrasen und ihre polnischen Entsprechungen. Eine konfrontative Studie. Frankfurt a.M.: Lang.

Witwicka-Iwanowska, Magdalena (2012): Artikelgebrauch im Deutschen. Eine Analyse aus der Perspektive des Polnischen. Tübingen: Narr.

Zifonun, Gisela/Hoffmann, Ludger/Strecker, Bruno (1997): Grammatik der deutschen Sprache. 3 Bde. Berlin/New York: de Gruyter. 
